\title{
Seroprevalence of Antibodies against SARS COV- 2 among Health Care Workers at Tertiary Care Hospital, Uttarakhand: A Retrospective study
}

\section{Mr. Amit K Saini}

All India Institute of Medical Sciences, Rishikesh, Uttarakhand, India

\section{Dr. Prasan Kumar Panda}

All India Institute of Medical Sciences, Rishikesh, Uttarakhand, India

\section{Dr. Yogesh Arvind Bahurupi}

All India Institute of Medical Sciences, Rishikesh, Uttarakhand, India

\section{Dr.Balram Omar}

All India Institute of Medical Sciences, Rishikesh, Uttarakhand, India

\section{Mr. Akhil T}

All India Institute of Medical Sciences, Rishikesh, Uttarakhand, India

\section{Ms. Pooja Panwar}

All India Institute of Medical Sciences, Rishikesh, Uttarakhand, India

Mr. Maneesh Sharma ( $\triangle$ manishsharma740@gmail.com )

All India Institute of Medical Sciences, Rishikesh, Uttarakhand, India

\section{Research Article}

Keywords: Seroprevalence, SARS COV-2, antibodies, health care workers

Posted Date: August 6th, 2021

DOl: https://doi.org/10.21203/rs.3.rs-783686/v1

License: (a) This work is licensed under a Creative Commons Attribution 4.0 International License.

Read Full License 


\section{Seroprevalence of Antibodies against SARS COV- 2 among Health Care Workers at Tertiary Care Hospital, Uttarakhand: A Retrospective study}

\section{ABSTRACT}

Background: The number of confirmed SARS-CoV-2 infections is vastly underestimated. In this context, Seroprevalence surveys are of utmost importance to assess the proportion of the population that has already developed antibodies against the virus and might potentially be protected against subsequent infection. Health care workers face greater risk of developing SARS-CoV-2. Therefore, present retrospective study was undertaken to estimate the prevalence of antibodies against SARS-CoV-2 among healthcare workers at tertiary care institute, Uttarakhand. Material and Methods : Data was gathered from Hospital records of 704 healthcare workers admitted at COVID 19 Unit and attended Covid OPD of tertiary care institute, Uttarakhand in-between 15th July to 14th Aug 2020. Result: Out of the 704 recruited participants, 14 (1.99\%) were seropositive for IgG antibodies against SARS-CoV-2. The cumulative prevalence of SARS-CoV-2 infection (presence of antibodies or past or current positive RT-PCR) was $4.40 \%$. Conclusion: The present study shows a low prevalence of SARS-CoV-2 IgG antibodies among health care workers. In addition, posting in COVID-19-positive areas was not associated with increased seropositivity. More studies are warranted to assess IgG/IgM antibodies against SARS -CoV2 among those HCW who are exposed to COVID-19 patients.

Keywords: Seroprevalence, SARS COV-2, antibodies, health care workers

\section{INTRODUCTION}

In December 2019, a novel viral disease caused by severe acute respiratory syndrome coronavirus emerged in Wuhan, China (1). After its emergence; it rapidly spread to the whole world and posed a global threat to public health. On 11th March 2020, WHO declared COVID-19 as a pandemic considering the severity of disease, morbidity, and mortality. Initial clinical manifestations of COVID-19 include fever, cough, headache, diarrhea, shortness of breath, nausea, vomiting, and fatigue(2). However, the clinical syndrome may vary from mild symptoms to severe pneumonia, acute respiratory distress, 
and even death(3). According to WHO's reports, globally, there were $12,964,809$ cases of COVID-19 and 570,288 deaths until 14th July 2020. In the Southeast Asia region, there were $1,196,651$ cases and 29,900 deaths by 14 th July 2020 . In India, the total numbers of cases reported were 906,752, and fatalities were 23,7272. In Uttarakhand, the total cases reported till 15th July 2020 were 3785, and deaths were 50(4).

The coronavirus disease-19 (COVID-19) pandemic, caused by severe acute respiratory syndrome coronavirus 2 (SARS CoV-2), a highly transmissible virus, has placed unprecedented strain on healthcare services worldwide and surpassed the other recent pandemics caused by SARS and Middle East Respiratory Syndrome (MERS) CoV(5). SARS $\mathrm{CoV}-2$ infection is diagnosed by directly identifying viral RNA or antigens, which is considered a gold standard for active disease. Indirect identification of SARS-CoV-2 infection identifies the previous contact with the virus by detecting specific anti-SARS-CoV2 antibodies(6). Three main types of antibodies are produced in response to infection; IgA, IgG, and IgM. IgM rises soonest and typically declines after infection. IgG and IgA persist and usually reflect a more extended longer-term immune response. Antibody tests look for variations in the above antibodies, either as a separate or combined antibody measurement. Antibody tests can be done in laboratory settings using enzyme-linked immunosorbent assays or chemiluminescence immunoassays (CLIA), using venous blood samples. The level of IgM antibody begins to rise after one week after the initial infection(7). At the same time, the IgG appears later than IgM (usually in 14 days after infection) and can last for six months or even several years, which means that the IgG serves as an indicator of the previous infection. SARS-CoV-2 can be transmitted through indirect, direct, or close contact with the infected person through oral/respiratory secretions or droplets. Airborne transmission of SARS-CoV-2 can occur by disseminating droplet nuclei (aerosols) that remain infectious when suspended in air over long distances and time(8).

Health care services are facing a significant burden because of the COVID -19 pandemic. Health care workers (HCW) are the frontline workforce directly or indirectly involved in the clinical care of suspected and confirmed COVID-19 cases. Potential occupational 
exposures place healthcare workers (HCWs) at higher risk of acquiring SARS-CoV-2 infection, which in turn may serve as an essential source of infection for their families and other community members(9). Therefore, health care workers such as doctors, nurses, hospital attendants, lab technicians are exposed to a higher risk of acquiring the disease than the general population(10). To plan an adequate public health response for HCWs and anticipate the disease dynamics, the measurement of anti-SARS-CoV-2 antibodies is of utmost importance. Knowledge of the Seroprevalence of SARS-CoV-2 antibodies among HCWs is essential to understand the spread of COVID-19 among healthcare facilities and assess public health interventions' success. Thereby, the present retrospective observational study was planned to identify COVID-19 Seroprevalence among HCWs.

\section{Material and Methods}

This retrospective cross-sectional study was carried out at the tertiary care hospital, Uttarakhand, between 15th July and 14th Aug 2020. Data were extracted to investigate study participants from E-Hospital records and data available with the medical record department (MRD) by a pre-validated checklist. Hospital records of 704 health care workers admitted at COVID 19 Unit and attended COVID-19 OPD during this one month were extracted and analyzed. Data of various HCWs, including doctors, nurses, administrative staff, technical staff, and paramedical staff, including hospital attendan ts, sanitary workers, and security personnel, were included. The details of demographic characteristics, job descriptions, exposure to COVID-19, any related symptoms, prior comorbidities, details of previous COVID-19 RT-PCR tests done were collected through a checklist from the questionnaire filled by on-duty physicians during screening OPD visit or hospital admission of HCWs. Incomplete records or missing data were excluded from the final analysis of the study. The approval for conducting the research study and data retrieving from MRD was obtained from the Institutional Ethical Committee wide letter no AIIMS/IEC/21/18 and the competent authority of the hospital before initiating the study. RT-PCR and IgG antibody reports of all the health care workers were collected. 


\section{RNA extraction of virus}

A total SARS-CoV-2 antibody (both IgG and IgM) chemiluminescence immunoassay (CLIA) was used (ADVIA Centaur COV2T assay, Siemens AG, Munich, Germany). A $2 \mathrm{ml}$ of venous blood was collected in a serum separator tube under aseptic conditions and processed in ADVIA Centaur XPT (Siemens AG, Munich, Germany) at Robotic Core Clinical Laboratory manufacturer's protocol and institutional infection control guidelines. This COV2T assay detected the antibody to spike protein receptor-binding domain (S1RBD) on the surface of the SARS-CoV-2 virus, which binds the virus to the target cells by a distinct human receptor (ACE2) found in the lung, heart, multiple organs, and blood vessels. The principle of this assay was antigen sandwich binding immunoassay in which antibodies from patient samples bind to the preformed complex of streptavidin-coated microparticles and binotinylated SARS-CoV-2 recombined antigens. This complex initiated the chemiluminescence reaction, which measured as relative light units (RLUs). A direct correlation existed between the amount of SARS-CoV-2 antibodies present in the sample and the amount of RLUs measured by the analyzer. The results were expressed in index value and reported as reactive ( $\geq 1$ index; positive for SARS-CoV-2 antibodies) or nonreactive ( $<1$ index; negative for SARS-CoV-2 antibodies).

\section{Comparator/outcomes}

No comparators were used in present study. Point prevalence of seroprevalence was measured. Data was transferred into Microsoft Excel spreadsheet and analyzed using statistical package for social sciences (SPSS) version 23. Frequency and percentag e were used to analyze demographic and clinical variables. Chi-square and fisher exact test were used to determine the association of demographic and clinical variable with serum antibody level.

\section{RESULTS}

Data of 704 health care workers was extracted from the Hospital records and data available with MRD. Out of 704 participants' data of 140 participants was from in patient COVID 19 Unit and 564 participants was from COVID-19 OPD. There were total of 5 RTPCR 
reports that were inconclusive, therefore, excluded in analyzing prevalence and correlation with IgG antibodies. The mean age of the participants was $27.23 \pm 6.26$. The majority of (58.9\%) participants were male, while $41.15 \%$ were females. Only $12.4 \%$ of the total participants reported that they had done duties in COVID positive ward in the last 28 days. $41.3 \%$ of participants revealed travel history to containment zone in past 28 days, and contact with COVID positive patient within last 28 days was reported by $26 \%$ of participants. $7.8 \%, 12.6 \%$, and $7.1 \%$ of the health care worker reported fever, cough, and sore throat in the past 28 days. Only $2 \%$ of HCW reported the presence of comorbid conditions (Table 1).

Table : 1 Symptoms experienced by HCWs in past 28 days $(n=704)$

\begin{tabular}{|l|c|c|}
\multicolumn{1}{|c|}{ Symptoms } & Frequency & Percentage (\%) \\
\hline Fever & 55 & 7.81 \\
\hline Headache & 3 & 4.26 \\
\hline Throat pain & 89 & 12.64 \\
\hline Cough & 50 & 7.10 \\
\hline Breathlessness & 1 & 0.14 \\
\hline Running nose & 28 & 3.97 \\
\hline Loss of taste/smell & 1 & 0.14 \\
\hline Diarrhea & 10 & 1.42 \\
\hline Abdominal pain & 5 & 0.71 \\
\hline Bleeding tendency & 3 & 0.42 \\
\hline Presence of any comorbidity & 14 & 1.98 \\
\hline
\end{tabular}

Of the 704 participants, 14 were seropositive for IgG antibodies against SARS-CoV-2. On RT-PCR, total 17 HCWs were detected positive. The cumulative prevalence of SARS-CoV-2 infection (presence of antibodies or past or current positive RT-PCR) was 4.40\% among HCW's and a strong correlation was found between RTPCR report and IgG report (OR: 11.76; CI: 3.28-34.34) with $p$ value of 0.0001 (Table 2). 
Table 2. Prevalence of IgG and RTPCR positivity along with their correlation $(n=699)$

\begin{tabular}{|c|c|c|c|c|}
\multirow{2}{*}{$\begin{array}{c}\text { IgG } \\
\text { report }\end{array}$} & \multicolumn{4}{|c|}{ RTPCR Report } \\
\hline & \multicolumn{3}{|c|}{ Negative } \\
\cline { 2 - 5 } & \multicolumn{3}{|c|}{ Positive } & \multicolumn{3}{c|}{ (Percentage) } \\
\hline Positive & Frequency & (Percentage) & Frequency & $(1.8 \%)$ \\
\hline Negative & 15 & $(11.8 \%)$ & 12 & $(98.2 \%)$ \\
\hline Total & 17 & $(100.0 \%)$ & 682 & $(100.0 \%)$ \\
\hline & & & & \\
\hline
\end{tabular}

*Total 5 RT-PCR reports were inconclusive

Results depicted no significant association of gender of the participants with IgG positive report ( $p=0.74$ ). Similarly visit/duties in COVID positive ward were not significantly associated with IgG positive report ( $\mathrm{p}=0.458$ ). It was found that contact of participants with COVID positive patient was not significantly associated with the IgG positive report ( $p=0.404$ ). History of travel to COVID positive locality was also not significantly associated with positive IgG report of the subjects ( $\mathrm{p}=0.131$ ) (Table 3 ) 
Table 3: Association of selected variables with Seroprevalence positive test $(n=704)$

\begin{tabular}{|c|c|c|c|c|c|}
\hline Variable & & Frequency & Seropositive & Seronegative & p value \\
\hline Age $\left(\right.$ Mean \pm SD) ${ }^{\mathrm{a}}$ & & $3 \pm 6.26$ & 14 & 690 & 0.802 \\
\hline & Male & 415 & 5 & 410 & \\
\hline Gender b & Female & 289 & 9 & 280 & 0.74 \\
\hline COVID positive & Yes & 88 & 1 & 87 & \\
\hline ward in last 28 & & & & & 0.458 \\
\hline days $^{b}$ & No & 615 & 13 & 603 & \\
\hline Travel history to & Yes & 289 & 7 & 282 & \\
\hline any positive & & & & & 0.131 \\
\hline $\begin{array}{l}\text { locality in last } \\
\text { 28days }^{\text {b }}\end{array}$ & No & 415 & 7 & 408 & \\
\hline Contact history to a & Yes & 183 & 1 & 182 & \\
\hline $\begin{array}{l}\text { positive case in last } \\
\text { 28days }{ }^{\text {b }}\end{array}$ & No & 521 & 13 & 508 & 0.404 \\
\hline
\end{tabular}

a Independent $\mathrm{t}$ test, $\mathrm{b}$ Chi-square test, $p=0.05$ level of significance

\section{DISCUSSION}

HCWs are frontline personnel responsible for the clinical management of suspected or confirmed COVID-19 patients. They are at a higher risk for acquiring the disease and, if infected, pose a threat to fellow HCWs, to vulnerable patients, and society. Therefore, regular screening of HCWs for SARS-CoV-2 infection is necessary to identify asymptomatic cases and exposure trends and formulate hospital policy to curb infection in the hospital setting. In present study 1.98\% health care workers were detected positive for IgG antibodies against SARS- COV-2, which was lower than reported studies by Hossain A et al.(10) 8.6\% Seroprevalence positive rate of IgG antibodies. Studies conducted by 
Madhusudan M et al.(6) , Gupta R et al.(11) and Mishra M et al.(7) reported 16.8\%, 13\% and $10.9 \%$ seropositivity to SARS-CoV2 among HCWs. While a study conducted by Anand S et al.(12) among dialysis patients in united states sero-prevalence was showed as 8\%. In contrary study conducted by Murhekar MV et al.(13) on SARS -CoV-2 antibody

Seroprevalence in India, stated that among the laboratory confirmed COVID-19 cases 81\% of patients were having SARS-CoV-2 IgG antibodies which is significantly higher in patients compared to HCW's in our study. Comparison of serosurveillance data between HCWs and National Centre for Disease Control (NCDC) by MoHFW June 2020, showed significantly higher Seroprevalence in the community than in HCWs at our institute(14). The lower seropositivity of COVID-19 infection amongst HCWs compared with other places could be due to effective and vigorous training and awareness by hospital administration, effective implementation of infection control practices in institute, also availability and righteous use of PPE, availability of rapid diagnostic tests for early identification of cases and prompt screening associated with contact tracing and quarantine. Posting in COVID-19-positive wards, travel history to any positive locality, and contact history to a positive case in past 28 days was not associated with increased seropositivity in HCWs, suggesting that the isolation protocols, infection control practices, and proper use of PPE were sufficient to prevent the spread of transmission to HCWs. In same line study conducted by Gupta R et al. (11) revealed that posting in COVID-19-designated wards and ICU was not associated with increased antibody positivity in HCWs. In contrary, study conducted by Rafi AM et al.(15) and Salim Khan M et al (9) contact with COVID 19 patient was found to be significantly associated with seropositivity (0.001) (0.014).

\section{LIMITATIONS}

The study was limited to health care workers. Only the records from 15 July to 14 August 2020 was extracted and analyzed.

\section{CONCLUSION}

We presented SARS-CoV-2 seroprevalence data in a broadly representative sample of HCW's working in tertiary care institute in Uttarakhand and showed a striking differences 
in seroprevalence by several HCW's characteristics, with lower seroprevalence among them. Also, testing of antibodies of the HCW's but the general public will help take better precautionary measures if tested positive. It will also help the administration in making a decision, developing new interventions and guidelines.

\section{References}

1. Zhu N, Zhang D, Wang W, Li X, Yang B, Song J, et al. A Novel Coronavirus from Patients with Pneumonia in China, 2019. N Engl J Med. 2020 Feb 20;382(8):727-33.

2. Patidar K, Sharma M, Gautam A, Sharma DK, Jain J. COVID-19 Knowledge and Perception among Budding Nurses: A Questionnaire-Based Survey'. International Journal of Nursing Research (IJNR). 2020;6(2):1-7. - Google Search [Internet]. [cited 2021 May 20].

3. Laboratory testing for coronavirus disease (COVID-19) in suspected human cases. WHO; [Cited March 19, 2020]. Available from: https://www.who.int/publicationsdetail/laboratory-testing-for-2019-novel-coronavirus-in-suspected-human-cases20200117 - Google Search [Internet]. [cited 2021 May 20].

4. COVID -19 pandemic in Uttarakhand.2021. Available from : https://en.wikipedia.org/wiki/COVID-19_pandemic_in_Uttarakhand\#July_2020. - Google Search [Internet]. [cited 2021 May 20].

5. Singh V, Supehia S, Gupta PK, Narula H, Sharma M, Devi K, Bhute AR. Effectiveness of video modules in infection control trainings during COVID 19 pandemic: A quasi experimental study in tertiary care institute. Journal of Education and Health Promotion. 2020;55:56. - Google Search [Internet]. [cited 2021 May 20].

6. Madhusudan M, Sankar J, Dhanalaxmi K, Putlibai S, Balasubramanian S. Seroprevalence to SARS-CoV-2 Among Healthcare Workers in an Exclusive Pediatric Hospital.2021. Indian pediatrics.vol. 58 - Google Search [Internet]. [cited 2021 May 20]. 
7. Mishra M, Chaudhry R, Rana F, Nag DS, Rai S. Serosurveillance of Health Care Workers in a COVID Hospital: Immune Response, and Its Longevity. Cureus [Internet]. 2021 Mar 21 [cited 2021 Jun 17];13(3).

8. World health organization: cornovirus update.(2021). Availble from : https://www.who.int/news-room/commentaries/detail/transmission-of-sars-cov-2implications-for-infection-prevention-precautions - Google Search [Internet]. [cited 2021 May 20].

9. Salim Khan M, Amin Qurieshi M. SARS-CoV-2 seroprevalence in healthcare workers of dedicated-COVID hospitals and non-COVID hospitals of District Srinagar, Kashmir. medRxiv [Internet]. 2020 Oct 27 [cited 2021 Jun 17];2020.10.23.20218164.

10. Hossain A, Nasrullah SM, Tasnim Z, Hasan MK, Hasan MM. Seroprevalence of SARS-CoV2 IgG antibodies among health care workers prior to vaccine administration in Europe, the USA and East Asia: A systematic review and meta-analysis. EClinicalMedicine. 2021 Mar $1 ; 33$.

11. Gupta R, Dwivedi T, Gajendra S, Sahoo B, Gupta SK, Vikas H. Policy Seroprevalence of antibodies to SARS-CoV-2 in healthcare workers \& implications of infection control practice in India. 2021;(March 2020):207-13.

\section{Anand S, Montez-Rath M, Han J, Bozeman J, Kerschmann R, Beyer P, Parsonnet J,} Chertow GM. Prevalence of SARS-CoV-2 antibodies in a large nationwide sample of patients on dialysis in the USA: a cross-sectional study. The Lancet. 2020 Oct 24;396(10259):133544. - Google Search [Internet]. [cited 2021 May 20].

13. Murhekar MV, Bhatnagar T, Selvaraju S, Saravanakumar V, Thangaraj JW, Shah N, Kumar MS, Rade K, Sabarinathan R, Asthana S, Balachandar R. SARS-CoV-2 antibody seroprevalence in India, August-September, 2020: findings from the second nationwide household serosurvey. The Lancet Global Health. 2021 Mar 1;9(3):e257-66. - Google Search [Internet]. [cited 2021 May 20]. 
14. Ministry of Health and Family Welfare, Government of India. Sero -prevalence study conducted by National Center for Disease Control NCDC, MoHFW, in Delhi, June 2020. Available from: https://pib.gov.in/PressReleasePage.aspx? PRID=1640137, accessed on November 16, 2020 - Google Search [Internet]. [cited 2021 May 20].

15. Mohamed Rafi A, Monica Lisa Joseph Tomy M, Thomas R, Valsan D, Unnikrishnan MG, Innah DJ, et al. Serosurveillance of SARS CoV 2 among the healthcare workers of a tertiary care teaching institution in Central Kerala during the post lockdown phase. medRxiv [Internet]. 2021 Jan 31 [cited 2021 Jun 17];2021.01.27.21250502. 